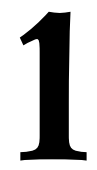

\title{
METAANÁLISIS DE LA INVESTIGACIÓN SOBRE MOOC EN EL PERÍODO 2013-2014
}

(META-ANALYSIS OF RESEARCH ON MOOC ALONG THE PERIOD 2013-2014)

\author{
Albert Sangrà \\ Universitat Oberta de Catalunya \\ Mercedes González-Sanmamed \\ Universidad de A Coruña \\ Terry Anderson \\ University of Athabasca
}

DOI: $10.5944 / e d u c X X 1.13463$

\section{Cómo referenciar este artículo/How to reference this article:}

Sangrà, A.; González Sanmamed, M. y Anderson, T. (2015). Metaanálisis de la investigación sobre mooc en el período 2013-2014. Educación XX1, 18(2), 21-49, doi: 10.5944/educXX1.13463.

Sangrà, A.; González Sanmamed, M. \& Anderson, T. (2015). Metaanálisis de la investigación sobre mooc en el período 2013-2014 [Meta-analysis of research on mooc along the period 20132014]. Educación XX1 , 18(2), 21-49, doi: 10.5944/educXX1.13463.

\section{RESUMEN}

El primer MOOC (Curso Masivo Abierto en Línea) se realiza en 2008 en Canadá. Desde entonces la proliferación de este tipo de iniciativas en diversos lugares del mundo ha suscitado interesantes controversias sobre sus aportaciones y significados en el ámbito del aprendizaje y la formación. Para superar los debates basados en opiniones, intuiciones o experiencias puntuales, es necesario disponer de evidencias que permitan una evaluación sistemática, detallada y contrastada de las posibilidades educativas de los MOOC.

Siguiendo la metodología utilizada en un estudio previo en el que se analizan las publicaciones realizadas durante los cinco primeros años, en este artículo se valoran las investigaciones desarrolladas en torno a los MOOC en el bienio 2013-14. Tras un riguroso proceso de revisión de la literatura disponible se identificaron 228 trabajos de investigación en estos dos últimos años.

Se presenta así un análisis cuantitativo y cualitativo de estas publicaciones clasificadas en función del año y tipo de publicación y once categorías temáticas propuestas. 
El estudio permite evidenciar una tendencia ascendente en el volumen de publicaciones y la predilección por artículos de revista y, en menor medida, por presentaciones en congresos. Las temáticas más investigadas han sido las referidas a valorar las estrategias pedagógicas y, especialmente, la motivación e implicación de los estudiantes.

El alcance del fenómeno MOOC para la docencia en línea está operando como un revulsivo que interpela tanto a las instituciones (su estructura, su modelo pedagógico, de gestión y de negocio) como a los agentes formadores (sus roles y sus competencias). Para que las respuestas que se deben ir construyendo puedan asentarse en las evidencias que tendrá que aportar la investigación, es necesario consensuar una agenda de los temas y las metodologías de estudio más relevantes.

\section{PALABRAS CLAVE}

MOOC; formación virtual; educación superior; cursos en línea; educación abierta; entornos de aprendizaje.

\section{ABSTRACT}

The first MOOC (Massive Open Online Course) was launched in 2008 in Canada. Ever since then the proliferation of these kinds of initiatives from all over the world have sparked interesting discussions regarding its benefits and implications in the field of education. In order to work through the debate surrounding MOOCs and their educational possibilities that have been generally based on opinion, intuition or isolated experiences it is necessary to have evidence that allows for a systematic, detailed and contrastive evaluation.

Following the methodology used in an earlier investigation that analyzed publications from the first five years, this article looks at studies that focus on MOOCs during the biennium of 2013-2014. Through a rigorous process of revision of the available literature, 228 investigative works from the last two years have been identified.

A quantitative and qualitative analysis of these publications is presented. Classification was based on the year of publication, the type of publication and eleven distinct proposed categories.

It has been observed that there is a rising tendency in the volume of publications and the predilection of journal articles and, to a lesser extent, presentations at conferences. Pedagogical strategies have been the most covered area as well as learner motivation and implication. 
The reach/scope of the MOOC phenomenon for online teaching has sparked and challenged both institutions (their structure, pedagogical model, management and business) as well as instructors (their roles and competencies). In order for answers to be able to settle in what the evidence in the investigation has been building, it is necessary to agree on a common set of topics and research methodologies.

\section{KEY WORDS}

MOOC; virtual education; higher education; online courses; open education; learning environments

\section{INTRODUCCIÓN}

MOOC es un acrónimo cuyas siglas en inglés significan curso masivo abierto en línea. Masivo porque pretende que el número de sus participantes sea lo más grande posible -mejor miles que cientos-, en contraposición a los cursos dirigidos a un número limitado de participantes. Abierto porque carece de restricciones y requisitos para acceder a él; porque utiliza recursos que no son propietarios y que están puestos a disposición de la comunidad, sin tener que pagar ninguna tasa de autoría por utilizarlos; y también porque es gratuito. Finalmente, en línea porque se lleva a cabo en la Red, en un contexto virtual, accesible desde cualquier lugar, en cualquier momento.

La primera aparición de un MOOC data del 2008 y se sitúa en Canadá. Su título era CCK08: Connectivism and Connective Knowledge (Conectivismo y Conocimiento Conectado), y sus autores fueron George Siemens y Stephen Downes. El curso se ofreció a los estudiantes que estaban matriculados en la Universidad de Manitoba, pero la novedad residió en que lo abrieron a todos aquellos que quisieran participar en el mismo, con el objetivo de poner de manifiesto el poder de las conexiones entre las personas a través de la Red para aprender y formarse. La única condición estuvo en que los profesores solo evaluarían a los estudiantes matriculados en la universidad. El resto, participaría de las actividades de autoevaluación y de evaluación entre pares para conocer su progreso en el aprendizaje de la materia. Superó las expectativas: se inscribieron más de 2.200 participantes.

En el año 2009, Sebastian Thrun y Peter Norvig, de la Universidad de Stanford, elaboraron el MOOC Introduction to Artificial Intelligence (Introducción a la Inteligencia Artificial), que convocó, según fuentes de la propia universidad, a más de 120.000 participantes de todo el mundo. Este MOOC, sin embargo, no tenía el mismo objetivo que el que desarrollaron Siemens 
y Downes. En este caso, el componente colaborativo cedía parte de su protagonismo al enfoque transmisivo, en el que destacaban los dos profesores de la universidad californiana.

Pero fue en 2012 cuando el fenómeno MOOC se convirtió en una tendencia. Pappano (2012) se hizo eco de ello en un reportaje en el New York Times titulado The Year of the MOOC (El año del MOOC). Este periódico sintetizaba los movimientos que se habían dado en el mercado educativo norteamericano durante ese año. Fundamentalmente, habían sido tres iniciativas empresariales las que habían alumbrado el uso de los cursos masivos abiertos y en línea: Coursera, edX y Udacity. Mientras que la primera se trataba de una joint venture con capital riesgo y se decidió por una estrategia eminentemente comercial y considerablemente agresiva; la segunda nació del acuerdo entre el Massachussets Institute of Technology (MIT) y la Universidad de Harvard, y se estructuró como una organización sin afán de lucro cuyo objetivo se manifestó como la voluntad de investigar en el campo del aprendizaje en línea. La tercera, Udacity, fue creada por Sebastian Thrun -ya citado anteriormente-, como una spin-off de la Universidad de Stanford con finalidades comerciales. En España, impulsada por Telefónica, el Banco de Santander y Universia, apareció MiríadaX, con el objetivo de convertirse en la plataforma MOOC en lengua castellana.

El auge de la educación en línea, sin embargo, no es un hecho puntual, ni se desencadena por la creación de algunos de estos MOOC. Los informes anuales impulsados por el Babson College (y antes por la Sloan Foundation) demuestran que la educación en línea ha ido aumentando en los Estados Unidos entre un 12 y un $20 \%$ en los últimos 7 u 8 años (Allen y Seaman, 2013). Prácticamente todos los estudiantes universitarios norteamericanos han tomado al menos un curso en línea durante su estancia en la universidad, y la previsión es que este dato vaya en aumento. Sin embargo, un aspecto fundamental en esta explosión reciente se puede observar en el comportamiento de las llamadas universidades top-tier, las punteras. Universidades que han ignorado la existencia de la educación en línea durante los últimos quince años, y que ahora la consideran como un elemento estratégico para su desarrollo futuro. El aumento de los costes de la educación superior en Estados Unidos ha contribuido a que algunas instituciones crean que la educación en línea puede ser la solución para abaratar dichos costes, aunque ese enfoque siempre ha generado serias dudas, según se desprende de diversos estudios (Rumble, 2004; Daniel, Kanwar y Uvalic-Trumbic, 2009; Bates y Sangrà, 2011; Contact North, 2014).

El año del MOOC generó una considerable cantidad de literatura, en especial en revistas y periódicos. Fundamentalmente, posicionamientos a favor y en contra de los MOOC habitualmente basados en intuiciones y 
opiniones de expertos, o en presentaciones de experiencias puntuales que se habían llevado a cabo con mayor o menor éxito, pero con poca o ninguna evaluación sistemática. Sin embargo, esto permitió que se establecieran interesantes discusiones que, más que dar respuestas, acumularon un considerable número de preguntas por responder. A continuación se expone una breve síntesis de las temáticas que merecieron -y en algunos casos, aún merecen - la atención de los debates establecidos.

Una cuestión en la que hay unanimidad es en que este fenómeno ha puesto la educación en línea en el escaparate mediático. Mientras que diversas instituciones están ofreciendo no solo cursos sino programas acreditados completamente en línea desde hace 20 años, muchas otras han descubierto el potencial de la educación en línea hace solo uno o dos años.

La filosofía abierta y la ausencia de restricciones para acceder a estos cursos, más allá de la obvia necesidad de disposición de las comunicaciones y dispositivos necesarios, pueden ayudar a democratizar el acceso a determinado conocimiento y hacerlo llegar a rincones donde la educación es un bien escaso (Rizvi, Donnelly y Barber, 2013). Sin embargo, otros sostienen que esta pretendida democratización es inexistente, puesto que los que cursan MOOC son, mayoritariamente, personas con formación universitaria y de países desarrollados (O’Shea et al., 2013).

Otro aspecto se refiere a la función que estos cursos pueden cumplir en las instituciones de educación superior. Cabe analizar más profundamente las dimensiones comerciales, económicas y de estrategia institucional que puede haber detrás de este fenómeno, además de la propiamente vinculada a la calidad del aprendizaje. La voluntad de expansión a otros públicos y a otros mercados y las estrategias de internacionalización, que persiguen también una mayor expansión en tiempos económicamente críticos, han hecho que algunas instituciones consideren los MOOC como un buen instrumento de carácter divulgativo de las propias organizaciones.

Por otro lado, y a pesar de que la masificación nunca ha sido una característica de la formación de éxito, los defensores de los MOOC abrazan este concepto porque consideran que la disponibilidad de datos de miles de personas desarrollando procesos de aprendizaje puede ayudar a conocer más y mejor cómo las personas aprenden. Esta creencia ha impulsado el concepto de analíticas de aprendizaje (Campbell, DeBlois y Oblinger, 2007).

La utilización de MOOC en una determinada universidad y su grado de controversia oscilará desde una posición menos controvertida cuando quiera usarse con finalidades comerciales (de captación de estudiantes, de 
publicidad, etc.) hacia una posición más discutida si quiere representar una disrupción en el campo pedagógico (Feldstein, 2014).

Aunque es posible que haya otros modelos menos conocidos, al hablar de MOOC, debemos tener en cuenta que existen dos grandes tipologías que centran la atención mediática. Los cMOOC y los xMOOC. Los primeros siguen los principios del conectivismo (Siemens, 2004), que considera que el aprendizaje se genera en red, a partir del contacto y de las relaciones que se establecen entre los nodos de esa red. Arguye que el conocimiento creado en grupo es mayor y más poderoso que el que se crea individualmente. Los cMOOC ponen recursos al alcance de aquellos que quieran compartirlos y sugieren generar interacciones con iguales para ampliar el conocimiento.

Los xMOOC disponen de una estructura mucho más simple. Su enfoque replica los métodos docentes más tradicionales: un conjunto de recursos, habitualmente audiovisuales - en la mayoría de los casos grabaciones de lecciones en clase-, puestos a disposición de quien quiera utilizarlos, y siempre con la finalidad de transmitir contenidos, a los que se añaden actividades de autoaprendizaje para quien quiera llegar al final del curso. La retroalimentación, habitualmente, está automatizada, y la evaluación se lleva a cabo mediante tests objetivos automáticos.

Por lo tanto, no se puede hablar de MOOC de forma unívoca. Algunas voces en el debate se refieren al diseño educativo de los MOOC como un factor clave de su supuesto éxito (Guàrdia, Maina y Sangrà, 2013; Conole, 2014; Ramírez, 2014). Sin embargo, lo cierto es que ninguno de estos modelos ha desarrollado aún mecanismos suficientemente eficientes para conocer cuál es el aprendizaje que sus participantes han alcanzado al final del proceso, lo cual tendría que ser el objetivo fundamental de este tipo de recursos puestos al alcance de quien quiere aprender.

Por otro lado, y aunque alguna de las críticas a los MOOC menciona la interpelación que se hace a la vanidad del profesor que será mundialmente conocido mediante los vídeos que de él o ella van a circular por toda la Red, también hay profesores entusiastas que están viendo en esta iniciativa una oportunidad para cambiar sus métodos docentes (Daniel, 2012): para enseñar mejor, para hacer que sus alumnos aprendan más y mejor, para ofrecerles más oportunidades de aprendizaje. En resumen, para experimentar.

Otro de los puntos de conflicto ha sido, y es, el éxito de los participantes. Por supuesto que cualquier definición de éxito será discutible. Y es lícito que sea así. Sin embargo, el verdadero problema es que, hasta ahora, sólo ha sido noticia cuántos estudiantes se inscriben en un MOOC, pero no cuántos terminan. El porcentaje de abandono en los MOOC es muy elevado. Mucho mayor 
que en otros modelos de educación en línea. La mayoría de autores sitúan este porcentaje de abandono entre el $87 \%$ (Onah, Sinclair y Boyatt, 2014) y el $95 \%$ (Ho et al., 2014), pasando por el 90\% (Rivard, 2013).

Hay estudios que demuestran que después de la primera semana, superado el efecto llamada o de novedad, el índice de participación y de colaboración entre estudiantes desciende dramáticamente, y en algunos casos pasa a ser inexistente (Lin, 2013).

Ante estos datos, algunos autores manifiestan que el porcentaje de abandono no importa (Wright, 2014), o que muchos solo quieren aprender, y que cuando ya saben lo que quieren, abandonan (Devlin, 2013). Es posible. Aunque entonces puede que debamos hablar más de divulgación que de formación. O quizás los MOOC se adecúan mejor a determinados perfiles de estudiantes autodidactas, que prefieren y son capaces de alcanzar sus objetivos formativos sin ninguna ayuda específica. En cualquier caso, suscita la duda sobre si realmente alcanzan sus objetivos o no. El sistema de evaluación en línea de tipo tradicional, con preguntas objetivas de respuesta múltiple, que es el mayoritariamente utilizado, no ayuda mucho a saber qué competencias el participante ha adquirido finalmente.

Finalmente, otro motivo de debate, no menos importante, es el modelo de negocio que puede permitir que los MOOC sean sostenibles. Sin duda, el hecho de que se ofrezcan de manera gratuita ha tenido mucho que ver en la percepción positiva que muchas personas tienen de ellos. Aplicando la teoría de la innovación disruptiva de Clayton Christensen (Christensen, Johnson y Horn, 2008), la aparición en el mercado de una oferta que rompe la estructura de costes existente (matrículas y tasas universitarias) y ofrece un producto que, aunque de calidad probablemente menor, es mucho más asequible para la población, podría llegar a ser la más aceptada.

Sin embargo, los modelos económicos de los MOOC no están nada claros, ni siquiera para aquellas empresas que se han lanzado a invertir en este campo. Algunos autores han concluido que se están experimentando modelos diversos para ver cuál de ellos puede resultar favorable (Haché y Punie, 2012; Pedreño et al., 2013)

Es evidente que todas las cuestiones comentadas anteriormente han sido tenidas en cuenta por las universidades a la hora de decidir cuál era su posición respecto de los cursos masivos abiertos y en línea. El estudio llevado a cabo por Hollands y Thirtali (2014) ha sintetizado muy bien cuáles pueden ser las razones por las cuales las instituciones de educación superior han abrazado con una efusividad poco habitual el fenómeno de los MOOC: 
- Ampliar el alcance de la institución y el acceso a la educación.

- Construir y mantener una marca.

- Mejorar la economía mediante la reducción de costes o el aumento de los ingresos.

- Mejorar los resultados educativos, tanto para los participantes en los MOOC como para los estudiantes presenciales.

- Innovar en el proceso de enseñanza y aprendizaje.

- Llevar a cabo investigación sobre la enseñanza y el aprendizaje.

Este último punto es el que resulta más relevante para los objetivos de nuestro estudio. Hasta el momento, la mayor parte del debate sobre los MOOC se ha movido en un marco de opiniones, favorables o desfavorables, que no se han basado en las evidencias. Algunas experiencias puntuales, tampoco han sido sujetas a una evaluación rigurosa y contrastada.

Sin embargo, es el momento de la investigación y de la valoración de los resultados que esta nos pueda facilitar. Los elementos que antes se han considerado como piezas del debate han generado, como ya se ha dicho, muchas más preguntas que respuestas: ¿Cuál es el nivel de profundidad del aprendizaje que se alcanza con los MOOC? ¿Existe un modelo de negocio sostenible que permita ofrecer cursos en línea de calidad completamente gratuitos? Si existe ese modelo, ¿para quién es sostenible y quién se beneficia del mismo? ¿Cuáles son las innovaciones pedagógicas que aportan los MOOC? ¿Cómo podemos saber realmente que los participantes en un MOOC alcanzan sus objetivos personales? ¿Qué significa calidad en un MOOC? Es, sin duda, a través de la investigación que podremos dar respuesta a estas preguntas.

En 2012, Liyanagunawardena, Adams y Williams (2013) realizaron una revisión sistemática de la literatura que se había publicado hasta aquel momento en referencia al fenómeno MOOC. Sin embargo, lo publicado en los dos años subsiguientes supera, en mucho, lo que se analizó en los 4 años anteriores. Por este motivo, se ha considerado muy importante la realización de una nueva revisión, que vaya más allá de las opiniones y de la presentación de experiencias puntuales, y se concentre en aquellas publicaciones que desvelan conclusiones que puedan dar más luz sobre el verdadero significado y potencial de este tipo de cursos. 
En este artículo se presenta un metaanálisis de la literatura correspondiente al bienio 2013-2014 que supone un avance respecto al realizado por los autores citados de 2008 al 2012, concentrándose en especial en las publicaciones que exponen resultados de investigación alrededor de la temática MOOC.

\section{MÉTODO}

La revisión de literatura que exponen Liyanagunawardena, Adams y Williams (2013) ha seguido el criterio de Fink (2010) para que otros puedan replicar sus métodos de identificación de trabajos susceptibles de ser considerados.

Este artículo toma como punto de partida dicha metodología, que consiste en identificar trabajos relevantes para la revisión en curso a partir de la indagación en revistas reconocidas en el campo, bases de datos, motores de búsqueda y otras revisiones sistemáticas a que se pueda tener acceso. Por lo tanto, y siguiendo su ejemplo, se buscaron resultados a partir del establecimiento de las mismas palabras clave utilizadas en el estudio de referencia:

- MOOC

- Massively Open Online Course

- Massive Open Online Course

Para evitar los problemas con que Liyanagunawardena, Adams y Williams (2013) se encontraron (como, por ejemplo, que de la revisión de determinadas revistas destacadas en el campo solo una de ellas dispusiese de artículos sobre la temática y que ninguno de ellos fuera relevante), se optó por empezar la búsqueda en las bases de datos académicas: ISI Web of Knowledge, ProQuest (ERIC, British Education Index and Australian Education Index), JSTOR (Education titles), IEEEXplorer y SCOPUS. Igual que en el estudio que se toma de referencia, algunos de los artículos se rechazaron porque no respondían al campo temático de este estudio, aunque el acrónimo coincidiese.

El criterio de relevancia consistió en seleccionar únicamente aquellos trabajos que presentasen un estudio empírico sobre los MOOC, rechazando artículos teóricos, informes políticos y position papers, así como aquellos que solamente planteasen una opinión o intuición sobre el fenómeno en cuestión. 
Finalmente, se aplicó también la técnica de encadenamiento de Gao, Luo y Zhang (2012), a partir de la cual pueden añadirse algunos trabajos que son citados de manera destacada en la literatura estudiada y que se incorporan al análisis general.

El proceso de recopilación de estas publicaciones concluyó con la identificación de un total de 228 trabajos, correspondientes al año 2013 y a los 9 primeros meses de 2014 .

El proceso de análisis desarrollado fue tanto cuantitativo como cualitativo. El análisis cuantitativo consistió en la clasificación de los trabajos de acuerdo al año de publicación y al tipo de publicación en el que aparecían, así como a su clasificación por categorías. Para el análisis cualitativo, y dado que lo que se pretendía era identificar las temáticas de investigación en que se centraban los estudios, se utilizaron y adaptaron las categorías que Hollands y Tirthali (2014) emplearon para identificar los ámbitos en los cuales la investigación sobre MOOC parece estar avanzando y sobre la cual manifiestan interés los responsables de las instituciones que fueron entrevistados en su estudio.

Las categorías iniciales fueron las siguientes:

1. El papel de las redes sociales en la enseñanza y el aprendizaje.

2. Poner a prueba estrategias pedagógicas.

3. Motivación e implicación de los estudiantes.

4. Investigación sobre modelaje/aprendizaje automático.

5. Procesamiento de lenguaje natural.

6. Interacción persona-ordenador.

7. Aprendizaje personalizado/adaptativo.

8. Comparar cursos híbridos con otros tradicionales.

9. Minería de datos educativa, analíticas de aprendizaje. 
Los autores de este estudio, al observar que algunos trabajos no encontraban una categoría adecuada a su naturaleza, añadieron dos categorías más:

10. Objetivos de las instituciones, consecuencias para el sistema de educación superior.

11. Cuestiones culturales y de accesibilidad.

\section{RESULTADOS}

Para la presentación de los resultados se decidió seguir dos perspectivas: cuantitativa y cualitativa. Desde un punto de vista cuantitativo, se ofrece una panorámica de los trabajos que se han encontrado teniendo en cuenta varios criterios de clasificación: concretamente, el año en que fueron publicados, la tipología de la publicación y la temática a la que se refieren en función de las categorías que se han considerado en la revisión que se realizó a partir del análisis de los estudios encontrados. Si bien en este último caso, se combinaron la perspectiva cuantitativa y cualitativa en el sentido de que la ubicación de los trabajos en las categorías - y, más aún, la definición de las propias categorías - ha seguido un proceso de análisis de contenido y de aproximación y revisión en la línea de los enfoques cualitativos. En definitiva, la cuantificación se ha realizado a posteriori de la definición de las categorías y del proceso de distribución de las publicaciones en dichas categorías. Hay que advertir, también, que algunos de los trabajos - al tener en cuenta los contenidos que abordaban- se han colocado en más de una categoría.

Tabla 1

Trabajos por año de publicación

\begin{tabular}{cc}
\hline Año & n. $^{\text {o }}$ de trabajos \\
\hline 2013 & 87 \\
2014 & 141 \\
\hline Total & 228
\end{tabular}

Tal y como ya se ha explicado anteriormente, esta revisión se circunscribe a los años 2013 y 2014. En la Tabla 1 se aprecia la distribución por año de publicación: en el año 2013 se encuentran 87 trabajos de investigación sobre MOOC y en el año 2014 la cifra asciende considerablemente y llega a 141 estudios. Si se tiene en cuenta el trabajo precedente que se tomó como referente para elaborar la presente revisión, la tendencia ascendente en cuanto al número de publicaciones que se constataba en 2012, continúa y se incrementa en los dos años siguientes. Como era de esperar tras la progresión advertida 
por Liyanagunawardena, Adams y Williams (2013), el fenómeno MOOC ha suscitado un gran interés en la comunidad científica y el crecimiento exponencial en cuanto a publicaciones es un claro reflejo del entusiasmo que ha despertado y de la fascinación que diversos agentes han encontrado en una iniciativa que aún provoca muchas dudas respecto a las posibilidades de negocio y de éxito que los Cursos Masivos Abiertos en Línea pueden generar. Concretamente, Liyanagunawardena, Adams y Williams (2013), identificaron un trabajo en 2008 y otro en 2009, siete en 2010, diez en 2011 y 26 en 2012. Es decir, entre el año 2012 y 2013 se triplicaron las publicaciones sobre MOOC y en dos años (de 2012 al 2014) se quintuplicaron.

Además, hay que advertir que las publicaciones que recopilaron Liyanagunawardena, Adams y Williams (2013) no eran estrictamente de investigación sino sobre cualquier aspecto relacionado con los MOOC. De ahí que una de las conclusiones que habría que reseñar es no solo la relevancia que han alcanzado de forma tan rápida y expansiva los MOOC, sino que además hayan suscitado estudios empíricos que permitan revisar sus aportes y contar con evidencias de lo que pueden significar en el campo de la formación en línea y de las condiciones óptimas para que su diseño y desarrollo resulte más eficaz (Guàrdia, Maina y Sangrà, 2013; Conole, 2014; Ramírez, 2014).

Tabla 2

Distribución de los trabajos por tipo de publicación

\begin{tabular}{|c|c|c|c|}
\hline Código & Tipo & $\mathbf{n}$ & $\%$ \\
\hline A & Artículos & 112 & 49,12 \\
\hline B & $\begin{array}{l}\text { Comunicaciones } \\
\text { congresos }\end{array}$ & 97 & 42,55 \\
\hline $\mathrm{C}$ & Periódicos & 4 & 1,75 \\
\hline $\mathrm{D}$ & Informes & 7 & 3,07 \\
\hline $\mathrm{E}$ & $\begin{array}{l}\text { Talleres (work- } \\
\text { shops) }\end{array}$ & 3 & 1,32 \\
\hline \multirow[t]{2}{*}{$\mathrm{F}$} & Libros/ensayos & 5 & 2,19 \\
\hline & Total & 228 & 100,00 \\
\hline
\end{tabular}

En la Tabla 2 se recoge el reparto de los trabajos en función del medio por el que se publicaron. Hay que destacar que casi la mitad de las publicaciones se difundieron a través de artículos de revista $(49,12 \%)$. Los artículos se encuentran repartidos en 48 revistas, de las cuales 26 son de acceso abierto y 22 de acceso restringido o de pago. De los 112 artículos revisados, 72 se han publicado en revistas de acceso abierto, por 40 en otras de acceso restringido o de pago. 
El segundo medio de difusión más utilizado ha sido mediante presentaciones en Congresos (42,55\%). Los siguientes sistemas de publicación se han utilizado de manera escasa: 3,07 \% en Informes; 2,19\% libros o ensayos; 1,75 en publicaciones periódicas de carácter divulgativo y 1,32\% en talleres o seminarios.

En cuanto a las publicaciones en revistas, conviene destacar que en varias de ellas se han organizado monográficos sobre MOOC y, en otras, está previsto que salgan a la luz en breve (concretamente, se prevé la publicación de un monográfico sobre MOOC en las revistas British Journal of Educational Technology (BJET), The International Review of Research in Open and Distance Learning (IRRODL), Journal of Universal Computer Science (JUCS), International Journal of Distance Education Technologies (IJDET) y Journal of Computer Assisted Learning (JCAL).

Sorprende que el número de trabajos difundidos en formato artículo supere al de presentaciones en Congresos, cuando habitualmente las reuniones científicas, cualquiera que sea su estructura organizativa (simposio, jornadas, encuentros,...), son mucho más ágiles y más inmediatas como escaparate de los últimos avances científicos y como medio para debatir y contrastar con colegas iniciativas más novedosas o en fase de experimentación. En este sentido es oportuno indicar que, en varios casos, los trabajos presentados en congresos fueron publicados en revistas (sobre todo en números monográficos) y quizás por esta razón se engrosó el recuento de trabajos en artículos de revista.

En estos dos años se han identificado siete informes, mayoritariamente elaborados o encargados por instituciones y organizaciones, cuya intención responde a la necesidad de sistematizar la información disponible para ofrecer una visión panorámica de los MOOC y, sobre todo, indagar en sus diversas implicaciones a nivel económico, académico, administrativo y pedagógico. Títulos tan significativos como los que se citan a continuación constituyen una muestra de los propósitos y el alcance de algunos de los informes que se han elaborado:

- MOOCs: expectations and reality. Full report (Center for Benefit-Cost Studies of Education Teachers College, Columbia University).

- An avalanche is coming (Institute for Public Policy Research).

- The pedagogy of the Massive Open Online Course: the UK view (The higher education academy).

- Structural limitations of learning in a crowd: communication vulnerability and information diffusion in MOOCs (Scientific Reports 4, Article number: 6447, Published 23 September 2014). 
- MOOCs@Edinburgh 2013 - report \#1 (https://www.era.lib.ed.ac. uk/handle/1842/6683).

En cuanto al número de libros publicados en estos dos años (2013 y 2014), si bien es escaso, resulta significativo si se tiene en cuenta que las publicaciones en formato libro exigen de mayor tiempo para su edición y difusión, y requieren de procesos más engorrosos para su aceptación por parte de las editoriales. De nuevo en estas publicaciones en formato libro se pone de manifiesto la preocupación de las instituciones de educación superior sobre los MOOC. Así, dos de las publicaciones, una en 2013 y otra en 2014, se han realizado bajo el patrocinio de la EUA (European University Association). También desde las universidades inglesas se ha editado en 2013 un libro sobre la temática de los MOOC: Massive open online courses. Higher education's digital moment? (London: Universities UK Publications).

Tabla 3

Distribución de los trabajos por año y tipo de publicación

\begin{tabular}{llrrrr}
\hline & & \multicolumn{2}{c}{$\mathbf{2 0 1 3}$} & \multicolumn{2}{c}{$\mathbf{2 0 1 4}$} \\
\hline Código & \multicolumn{1}{c}{ Tipo } & n & \multicolumn{1}{c}{$\%$} & $\mathbf{n}$ & \multicolumn{1}{c}{$\%$} \\
\hline A & Artículos & 37 & 42,52 & 75 & 53,19 \\
B & Comunicaciones & 40 & 45,98 & 57 & 40,42 \\
C & Periódicos & 4 & 4,60 & 0 & -- \\
D & Informes & 3 & 3,45 & 4 & 2,84 \\
E & Talleres (work- & 0 & -- & 3 & 2,13 \\
& shops) & & & & \\
F & Libros/Ensayos & 3 & 3,45 & 2 & 1,42 \\
\hline & Total & 87 & 100,00 & 141 & 100,00
\end{tabular}

Tal y como se observa en la Tabla 3, la clasificación de los trabajos según el año y la tipología de la publicación es bastante similar si se atiende a los porcentajes alcanzados en función de las distribución por cada categoría en cada periodo anual. De todas formas hay diferencias que, a pesar de ser mínimas, merece la pena comentar. En primer lugar, advertir del aumento significativo en cuanto a las publicaciones en artículos. Como ya se ha dicho, la publicación de un artículo - sobre todo en revistas indexadasestá sujeta a un proceso de revisión - y en muchos casos de corrección por parte de los autores-, que requiere de un tiempo que puede llevar meses o incluso un año. Esto quizás pueda ser una de las causas de la diferencia en el número de publicaciones encontradas entre ambos años. Esto, obviamente, además de la progresión general que se observa si se compara el año 2012 
con el 2014, y que se aprecia y repercute en todas las tipologías reseñadas excepto en las publicaciones periódicas de difusión (no hemos identificado ninguna en 2014).

El número de presentaciones a congresos también se ha incrementado y remarca la importancia del tema de los MOOC para los investigadores y estudiosos, como se refleja en los diversos eventos que se han celebrado en ambos períodos. Efectivamente, se puede advertir que los congresos que han incluido presentaciones de MOOC son de temática variada e incluyen tanto reuniones de índole más tecnológica o de gestión como aquellos otros de perspectiva más educativa. Como ejemplo de los dos primeros ámbitos se pueden citar los siguientes congresos:

- Advanced Learning Technologies (ICALT), 2014 IEEE 14th International Conference on (LOOCs -- Linked Open Online Courses: A Vision).

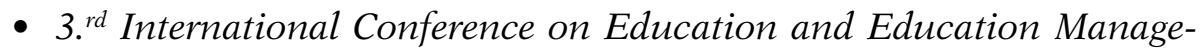
ment (EEM) Location: Singapore, SINGAPORE Date: NOV 15-16, 2013 (The Bilingual Teaching Reform Combined with MOOC).

Por su parte, los congresos de carácter educativo, priorizan los aspectos relativos al aprendizaje a través de los MOOC:

- Proceedings of the European Conference on e-Learning, ECEL (Something for everyone: MOOC design for informing dementia education and research).

- IADIS International Conference on Cognition and Exploratory Learning in Digital Age, CELDA 2013 (Developing a connectivist MOOC at a college of education: Narrative of disruptive innovation?).

Llama la atención que en 2013 no se contabilice ningún taller que incluya temáticas sobre MOOC y que de los tres trabajos desarrollados en 2014, dos de ellos se presentasen en el mismo evento: 3rd International Workshop on Learning Technology for Education in the Cloud, LTEC 2014; Santiago; Chile; 2 September 2014 through 5 September 2014; Code 10735.

Tabla 4

Distribución de los trabajos por categoría temática

\begin{tabular}{|c|c|c|c|}
\hline Código & Categoría & $\mathbf{n}$ & $\%$ \\
\hline 1 & $\begin{array}{l}\text { El papel de las redes sociales en la } \\
\text { enseñanza y el aprendizaje }\end{array}$ & 53 & 23,24 \\
\hline 2 & Poner a prueba estrategias pedagógicas & 84 & 36,84 \\
\hline 3 & Motivación e implicación de los estudiantes & 64 & 28,07 \\
\hline
\end{tabular}




\begin{tabular}{clrr}
\hline Código & \multicolumn{1}{c}{ Categoría } & n & \multicolumn{1}{c}{$\%$} \\
\hline 4 & Modelaje/aprendizaje automático & 16 & 7,02 \\
5 & Procesamiento del lenguaje natural & 15 & 6,58 \\
6 & $\begin{array}{l}\text { Interacción entre las personas y el ordena- } \\
\text { dor }\end{array}$ & 38 & 16,67 \\
7 & Aprendizaje personalizado & 16 & 7,02 \\
8 & $\begin{array}{l}\text { Comparar los cursos tradicionales con los } \\
\text { híbridos }\end{array}$ & 22 & 9,65 \\
9 & $\begin{array}{l}\text { Minería de datos educativa, analíticas de } \\
\text { aprendizaje }\end{array}$ & 43 & 18,86 \\
10 & $\begin{array}{l}\text { Objetivos de las instituciones, consecuen- } \\
\text { cias para el sistema de educación superior }\end{array}$ & 51 & 22,37 \\
11 & Cuestiones culturales y de accesibilidad & 16 & 7,02
\end{tabular}

Como se ha advertido anteriormente, las categorías utilizadas para clasificar temáticamente los trabajos que se encontraron a partir de la revisión bibliográfica, han surgido de un doble proceso que se puede clasificar como deductivo e inductivo: inicialmente se utilizaron las nueve categorías recogidas por Hollands y Tirthali (2014) y al aplicarlas para ordenar las publicaciones se vio la necesidad de ampliar dos categorías más para recoger aspectos particulares de la investigación que difícilmente podrían ubicarse en las nueve seleccionadas. De esta forma, el total de categorías con las que se trabajó fue de once.

También cabe recordar que algunos trabajos se ubicaron en más de una categoría teniendo en cuenta las temáticas que desarrollaban. De ahí que la suma total de las publicaciones reseñadas en la Tabla 4 supera los 228 trabajos que se han revisado (si bien los porcentajes se han calculado tomando como referencia los 228 estudios encontrados).

Los datos recogidos en la Tabla 4 permiten afirmar que más de un tercio de las investigaciones sobre MOOC se han orientado al análisis de las estrategias pedagógicas implementadas: por ejemplo, co-creación de contenido y pedagogía participativa (Andersen y Ponti, 2014); o diseño de MOOC para nivelación de conocimientos (Daza, Makriyannis y Rovira Riera, 2013). Más de la cuarta parte de los trabajos se centraron en el estudio del nivel de motivación y la implicación de los estudiantes: evaluación de sistemas de apoyo a los estudiantes (Stewart et al., 2013); razones para inscribirse en un MOOC y experiencia de aprendizaje (Zutzhi, O’Hare y Rodafinos, 2013); o estrategias para animar a la participación (Kizilcec et al., 2014). En un porcentaje similar de trabajos se intentó dar respuesta a dos de las temáticas 
de gran trascendencia social e institucional: En cuanto a la categoría uno, a explorar el papel de las redes sociales en la enseñanza: uso de herramientas 2.0 para el aprendizaje (Alario-Hoyos et al., 2014); o cómo se aprende colaborando en redes en línea y offline (Schreurs et al., 2014); y respecto a la categoría diez, identificar cómo se cubrían los objetivos de las instituciones y valorar las consecuencias para el sistema de educación superior: estrategias de internacionalización (Marshall, 2013); análisis económico y de sostenibilidad del sistema (Hoxby, 2014); o cambios en las dinámicas institucionales (O'Connor, 2014). Las siguientes categorías que han suscitado mayor interés indagatorio han sido la referida a la denominada minería de datos educativa y a las analíticas de aprendizaje $(18,86 \%)$ : análisis del uso de los recursos con respecto al tiempo utilizado en cada uno de ellos (Breslow et al., 2013); o estrategias de navegación en función de la edad y el país de origen (Guo y Reinecke, 2014) y la interacción entre las personas y el ordenador (16,67\%): adaptación de la máquina al progresos del estudiante (Vargas, 2014). Las siguientes categorías han sido menos inspeccionadas y el número de trabajos encontrados no alcanza en ningún caso el $10 \%$. Tal es la situación de las categorías relativas a la comparativa entre los cursos tradicionales y los híbridos, investigación sobre modelajelaprendizaje automático, el aprendizaje personalizado, así como las cuestiones culturales y de accesibilidad.

Tabla 5

Distribución de los trabajos por año y tipo de publicación

\begin{tabular}{|c|c|c|c|c|c|}
\hline \multirow[b]{2}{*}{ Código } & \multirow[b]{2}{*}{ Categoría } & \multicolumn{2}{|c|}{$2013^{1}$} & \multicolumn{2}{|c|}{$2014^{2}$} \\
\hline & & $\mathbf{n}$ & $\%$ & $\mathbf{n}$ & $\%$ \\
\hline 1 & $\begin{array}{l}\text { El papel de las redes so- } \\
\text { ciales en la enseñanza y el } \\
\text { aprendizaje }\end{array}$ & 16 & 18,39 & 37 & 26,24 \\
\hline 2 & $\begin{array}{l}\text { Poner a prueba estrategias } \\
\text { pedagógicas }\end{array}$ & 35 & 44,82 & 49 & 34,75 \\
\hline 3 & $\begin{array}{l}\text { Motivación e implicación } \\
\text { de los estudiantes }\end{array}$ & 19 & 21,84 & 45 & 31,91 \\
\hline 4 & $\begin{array}{l}\text { Modelaje/aprendizaje } \\
\text { automático }\end{array}$ & 5 & 5,75 & 11 & 7,80 \\
\hline 5 & $\begin{array}{l}\text { Procesamiento del lengua- } \\
\text { je natural }\end{array}$ & 6 & 6,90 & 9 & 6,38 \\
\hline 6 & $\begin{array}{l}\text { Interacción entre las per- } \\
\text { sonas y el ordenador }\end{array}$ & 19 & 21,84 & 19 & 13,47 \\
\hline 7 & Aprendizaje personalizado & 5 & 5,75 & 11 & 7,80 \\
\hline
\end{tabular}




\begin{tabular}{clcccc}
\hline & \multicolumn{2}{c}{$\mathbf{2 0 1 3}^{1}$} & \multicolumn{2}{c}{$\mathbf{2 0 1 4}^{2}$} \\
\hline Código & \multicolumn{1}{c}{ Categoría } & n & \% & n & \% \\
\hline 8 & $\begin{array}{l}\text { Comparar los cursos tra- } \\
\text { dicionales con los híbri- } \\
\text { dos } \\
9\end{array}$ & 12 & 13,79 & 10 & 7,09 \\
& $\begin{array}{l}\text { Minería de datos educa- } \\
\text { tiva, analíticas de apren- } \\
\text { dizaje }\end{array}$ & 13 & 14,94 & 30 & 21,28 \\
& $\begin{array}{l}\text { Objetivos de las institucio- } \\
\text { nes, consecuencias para } \\
\text { el sistema de educación } \\
\text { superior }\end{array}$ & 24 & 27,59 & 27 & 19,15 \\
& $\begin{array}{l}\text { Cuestiones culturales y de } \\
\text { accesibilidad }\end{array}$ & 4 & 4,60 & 12 & 8,51 \\
\hline
\end{tabular}

${ }^{1}$ El porcentaje se ha calculado considerando el total de las publicaciones realizadas durante el año 2013, es decir, 87 trabajos.

${ }^{2}$ El porcentaje se ha calculado considerando el total de las publicaciones realizadas durante el año 2014, es decir, 141 trabajos.

En la distribución de los trabajos por año y categoría temática, tal y como aparece en la Tabla 5 se aprecian algunas diferencias en varias de las categorías y también ciertas coincidencias.

Así, tanto en 2013 como en 2014, la investigación se concentra en el análisis de las estrategias pedagógicas (44,82\% en 2013 y 34,75\% en 2014). El segundo tema de mayor interés en 2013 fue el alcance de los MOOC en cuanto a los objetivos de las instituciones y sus consecuencias para el sistema de educación superior (27,59\% en 2013 y algo menor en 2014 con $19,15 \%)$. En 2014, la segunda temática sobre la que más se investigó fue sobre la motivación e implicación de los estudiantes (31,91\% en 2014 y algo menor en 2013 con $21,84 \%$ ).

En 2013, también despertaron interés temáticas como las posibilidades de interacción entre las personas y los ordenadores, así como la investigación sobre modelaje/aprendizaje automático $(21,84 \%)$. En este mismo año también se publicaron estudios acerca del papel de las redes sociales en el aprendizaje $(18,39 \%)$; la minería de datos $(14,94 \%)$ o la comparativa entre los cursos tradicionales y los híbridos $(13,79)$.

En 2014, se contabilizan un buen número de estudios acerca del papel de las redes sociales en el aprendizaje $(26,24 \%)$. Y se mantiene la 
preocupación por la minería de datos $(21,28 \%)$ y el análisis acerca de los objetivos de las instituciones y las consecuencias para el sistema de educación superior $(19,15 \%)$.

\section{Tabla 6}

Distribución de los trabajos por tipo de publicación y categoría temática

\begin{tabular}{|c|c|c|c|c|c|c|c|c|c|c|c|c|c|c|}
\hline \multirow[b]{2}{*}{ Cod. } & \multirow{2}{*}{$\begin{array}{c}\text { Tipo publicación } \\
\text { Categoría }\end{array}$} & \multicolumn{2}{|c|}{ Artículo } & \multicolumn{2}{|c|}{ Congreso } & \multicolumn{2}{|c|}{ Periódico } & \multicolumn{2}{|c|}{ Informe } & \multicolumn{2}{|c|}{ Taller } & \multicolumn{2}{|c|}{ Libro } & \multirow[b]{2}{*}{ Total } \\
\hline & & $\mathbf{n}$ & $\%$ & $\mathbf{n}$ & $\%$ & $\mathbf{n}$ & $\%$ & $\mathbf{n}$ & $\%$ & $\mathbf{n}$ & $\%$ & $\mathbf{n}$ & $\%$ & \\
\hline 1 & $\begin{array}{l}\text { Papel redes socia- } \\
\text { les en enseñanza } \\
\text { y aprendizaje }\end{array}$ & 32 & 60,37 & 17 & 32,07 & 1 & 1,89 & 1 & 1,89 & 1 & 1,89 & 1 & 1,89 & 53 \\
\hline 2 & $\begin{array}{l}\text { Poner a prueba } \\
\text { estrategias peda- } \\
\text { gógicas }\end{array}$ & 48 & 57,15 & 31 & 36,90 & 2 & 2,38 & 2 & 2,38 & 0 & --- & 1 & 1,19 & 84 \\
\hline 3 & $\begin{array}{l}\text { Motivación e } \\
\text { implicación de los } \\
\text { estudiantes }\end{array}$ & 34 & 53,13 & 25 & 39,06 & 1 & 1,56 & 0 & --- & 1 & 1,56 & 3 & 4,69 & 64 \\
\hline 4 & $\begin{array}{l}\text { Modelaje/aprendi- } \\
\text { zaje automático }\end{array}$ & 4 & 25 & 12 & 75 & 0 & --- & 0 & --- & 0 & --- & 0 & --- & 16 \\
\hline 5 & $\begin{array}{l}\text { Procesamiento } \\
\text { del lenguaje } \\
\text { natural }\end{array}$ & 8 & 53,33 & 5 & 33,33 & 0 & --- & 1 & 6,67 & 0 & --- & 1 & 6,67 & 15 \\
\hline 6 & $\begin{array}{l}\text { Interacción perso- } \\
\text { na-ordenador }\end{array}$ & 10 & 26,31 & 27 & 71,06 & 0 & --- & 0 & --- & 0 & --- & 1 & 2,63 & 38 \\
\hline 7 & $\begin{array}{l}\text { Aprendizaje } \\
\text { personalizado }\end{array}$ & 9 & 56,25 & 6 & 37,5 & 0 & --- & 0 & --- & 0 & --- & 1 & 6,25 & 16 \\
\hline 8 & $\begin{array}{l}\text { Comparar cursos } \\
\text { tradicionales con } \\
\text { híbridos }\end{array}$ & 12 & 54,55 & 7 & 31,83 & 1 & 4,54 & 1 & 4,54 & 0 & --- & 1 & 4,54 & 22 \\
\hline 9 & $\begin{array}{l}\text { Minería de } \\
\text { datos educativa, } \\
\text { analíticas de } \\
\text { aprendizaje }\end{array}$ & 11 & 25,59 & 28 & 65,12 & 0 & --- & 1 & 2,32 & 2 & 4,65 & 1 & 2,32 & 43 \\
\hline 10 & $\begin{array}{l}\text { Objetivos } \\
\text { institucionales; } \\
\text { consecuencias } \\
\text { educación su- } \\
\text { perior }\end{array}$ & 25 & 49,03 & 13 & 25,49 & 2 & 3,92 & 5 & 9,80 & 1 & 1,96 & 5 & 9,80 & 51 \\
\hline 11 & $\begin{array}{l}\text { Cuestiones } \\
\text { culturales y de } \\
\text { accesibilidad }\end{array}$ & 8 & 50 & 5 & 31,25 & 1 & 6,25 & 1 & 6,25 & 1 & 6,25 & 0 & --- & 16 \\
\hline
\end{tabular}

Se analizan seguidamente, en función de los resultados que se reflejan en la Tabla 6, cuáles han sido los formatos de publicación más utilizados para cada una de las once categorías temáticas que se han establecido. 
Más de la mitad de los estudios referidos al papel de las redes sociales en el aprendizaje se han publicado en artículos de revista (60,37\%). Un tercio de las investigaciones sobre esta línea de indagación se presentaron en congresos $(32,07 \%)$. Los demás medios de difusión han sido escasamente utilizados.

También más de la mitad de los estudios relativos a la valoración de las estrategias pedagógicas se pueden localizar en publicaciones de revista $(57,15 \%)$, aunque también han sido considerables las presentaciones en congresos sobre esta temática $(36,90 \%)$.

Los análisis sobre la motivación e implicación de los estudiantes se pueden encontrar en un alto porcentaje en artículos de revista $(53,13 \%)$ y en congresos $(39,06 \%)$. Cabe mencionar que un 4,69 de los estudios se recogieron en libros.

Tres cuartas partes de la investigación acerca del modelaje/aprendizaje automático se han presentado fundamentalmente en congresos y la cuarta parte restante en artículos. No se han encontrado estudios de esta naturaleza en los otros medios de difusión rastreados.

La mitad de las investigaciones sobre procesamiento del lenguaje natural se pueden consultar en artículos $(53,33 \%)$. Un tercio de los estudios se han presentado a congresos $(33,33)$.

Para consultar los análisis acerca de la interacción persona-ordenador hay que consultar, sobre todo, las publicaciones de congresos $(71,06 \%)$. Una cuarta parte de los estudios se han recogido en artículos de revista $(26,31 \%)$ y en mucha menor medida en libros $(2,63 \%)$.

Más de la mitad de los análisis acerca del aprendizaje personalizado $(56,25 \%)$ y sobre la comparativa entre los cursos tradicionales con los híbridos $(54,55)$ han elegido la publicación en revistas especializadas como medio de difusión. Pero tampoco es desdeñable la presentación de investigaciones sobre estos asuntos en congresos $(37,5 \%$ y $31,83 \%$, respectivamente)

Sobre minería de datos educativos y analiticas de aprendizaje, se han presentado investigaciones mayoritariamente en congresos $(65,12 \%)$, aunque también una cuarta parte se difundió a través de artículos de revista $(25,59 \%)$. Una de las razones por las cuales puede explicarse esta tendencia es el hecho de que el perfil de los autores de este tipo de investigaciones es el de ingenieros, en informática o en telecomunicaciones, que suelen diseminar los resultados de sus trabajos en forma de comunicaciones en congresos de su ámbito. Esta área de conocimiento valora mucho la presentación de 
este tipo de comunicaciones, puesto que la mayoría de actas de estos congresos se encuentran indexadas en bases de datos académicas de prestigio (i.e. ISI Web of Knowledge, ProQuest) que les dan gran difusión. Es más tarde cuando, eventualmente, se decide publicar otra versión del trabajo en forma de artículo de revista.

En cuanto a los estudios reseñados en las dos últimas categorías, relativos a los objetivos de las instituciones y las consecuencias para el sistema de educación superior, así como los análisis sobre cuestiones culturales y de accesibilidad: la mitad de ellos se han difundido en artículos de revista y una tercera parte se presentaron a congresos.

\section{DISCUSIÓN Y CONCLUSIONES}

El incremento de publicaciones que recogen trabajos empíricos y, particularmente, su destacada magnitud en los años 2013 y 2014, demuestra que se ha entrado en una nueva etapa en el fenómeno MOOC, orientada a buscar la explicación de sus significados y el contraste de los resultados que se pueden obtener en proyectos de investigación con rigor científico, en la línea de lo que ya exigían algunos autores (Bates, 2014; Veletsianos, 2014).

Los temas de investigación, que se han categorizado a partir de la clasificación de Hollands y Tirthali (2014), no concuerdan completamente con lo que los expertos esperan de una agenda para la investigación en MOOC. Así, Downes (2013) señala que la Fundación Bill y Melinda Gates promueve unas temáticas de estudio que tienen como puntos prioritarios: identificar el tipo de datos que es necesario obtener para promover el avance del aprendizaje; las finalidades y los diseños de los distintos tipos de MOOC; los tipos de estudiantes, cursos y contextos para los cuales los MOOC son efectivos; la profundidad del aprendizaje con MOOC; los componentes de los MOOC que más impactan en el aprendizaje de los participantes; los roles del profesor; las condiciones temporales, y las condiciones de trabajo distintas entre el aprendizaje tradicional y el que se lleva a cabo en línea; los costes y los obstáculos para su desarrollo; y los objetivos que tanto estudiantes como instituciones pueden ayudar a alcanzar a través de los MOOC.

Por su parte, Bates (2014) incide en destacar la importancia de indagar en qué es lo que aprenden los participantes en un MOOC, sus costes y las posibilidades de desarrollar economías de escala, el impacto en la marca institucional, las cuestiones éticas y la respuesta a las necesidades de los estudiantes en la era digital. 
Del trabajo realizado se desprende que, si bien algunos de estos aspectos sí que son contemplados en las distintas publicaciones analizadas, hay una parte muy importante de la investigación orientada al terreno de las soluciones tecnológicas (Modelaje/aprendizaje automático, procesamiento del lenguaje natural, interacción persona-ordenador, minería de datos y analíticas de aprendizaje).

Es muy probable que eso sea debido, por un lado, a que los xMOOC, (a los que ya se ha hecho referencia en este artículo y que son los mayoritarios en este momento) tienen su génesis en el ámbito de las ingenierías; y, por otro, a que los profesionales que tomaron iniciativas empresariales que impulsaron este fenómeno (Coursera, edX, Udacity) cuentan precisamente con el mismo perfil. De ahí que la mayoría de comunicaciones a congresos analizadas en el bienio que nos ocupa provengan de eventos en el área de la informática y las telecomunicaciones (Learning at Scale Interational Conference, Association of Computer Machinery (ACM); EDUCON IEEE International Conference).

Otro de los motivos que explicaría este hecho es que los modelos de negocio experimentados no garantizan la sostenibilidad de la iniciativa (Haché y Punie, 2012), con lo que existe una acuciante necesidad de encontrar propuestas que reduzcan drásticamente el coste de elaboración y mantenimiento de los MOOC, para lo cual la automatización del proceso docente, y la consiguiente reducción de la intervención de profesores se observa como una posible vía a explorar.

Sin embargo, cabe destacar que las temáticas más investigadas han sido las referidas a valorar las estrategias pedagógicas y, especialmente, la motivación e implicación de los estudiantes. Ahí, las críticas —incluso por parte de algunos de los mayores defensores de esta modalidad (Siemens, 2012) —, a la ausencia de innovación pedagógica de los xMOOC se han hecho bien patentes, al mismo tiempo que existe una gran preocupación por la falta de continuidad - y su consiguiente abandono- en el aprendizaje de la mayoría de participantes en estos cursos, ya sea por parte de aquellos que lo utilizan como evidencia para criticar el supuesto éxito de los MOOC, como por aquellos que quieren encontrar interpretaciones que les permitan seguir defendiendo su bondad (Devlin, 2013; Ho et al., 2014).

El estudio realizado ha puesto de manifiesto que existe una tendencia ascendente en el volumen de publicaciones y en la predilección por que estas tomen el formato de artículos de revista y, en menor medida, de comunicaciones en congresos. Cabe destacar el gran número de artículos que se publican en revistas en abierto, siguiendo la filosofía de este movimiento, del cual los MOOC serían una enseña paradigmática. Las revistas en abierto 
permiten una publicación más rápida y facilitan un mayor acceso a los lectores. Además, vistos los números monográficos que están en preparación (algunos de ellos ya se han mencionado), probablemente será conveniente llevar a cabo una nueva revisión de literatura en un tiempo prudencial para verificar y contrastar los avances que se vayan logrando.

Desde un punto de vista pedagógico, el alcance del fenómeno MOOC para la docencia en línea está operando, sin duda, como un revulsivo que interpela, por un lado a las instituciones en cuanto a su estructura, su modelo pedagógico, de gestión y de negocio. Pero por otro lado también tiene importantes implicaciones para la docencia y, en particular, la docencia en línea. Toma un cariz mucho más relevante el analizar y desarrollar los roles y las competencias que los docentes en línea deben asumir y adquirir para desempeñarse con éxito en entornos virtuales que, como se puede comprobar, son cambiantes (Muñoz-Carril, González-Sanmamed y HernándezSellés, 2013; González-Sanmamed, Muñoz-Carril y Sangrà, 2014).

La importante cantidad de investigación que genera este fenómeno exigirá que las respuestas que se deben construir, se asienten en las evidencias que se vayan encontrando, por lo cual, será necesario consensuar una agenda de los temas y las metodologías de estudio de mayor utilidad para que su impacto pueda ser adecuadamente aprovechado en beneficio del avance de una educación más abierta y para todos. 


\section{REFERENCIAS BIBLIOGRÁFICAS}

Alario-Hoyos, C.; Pérez-Sanagustín, M.; Delgado-Kloos, C.; Parada G., H. A. y Muñoz-Organero, M. (2014). Delving into Participants' Profiles and Use of Social Tools in MOOCs, IEEE Transactions on Learning Technologies, 7(3), 260-266.

Allen, I. E. y Seaman, J. (2013). Changing Course: Ten Years of Tracking Online Education in the United States. Wellesley, MA: Babson College/Quahog Research Group.

Andersen, R. y Ponti, M. (2014). Participatory pedagogy in an open educational course: challenges and opportunities, Distance Education, 35(2), 234-249.

Bates, A. W. (2014, Octubre 19). The strengths and weaknesses of MOOCs: Part I [blog post]. Recuperado de http://www.tonybates.ca/tag/moocs/

Bates, A. W. y Sangrà, A. (2011). Managing Technology in Higher Education. Strategies for Transforming Teaching and Learning. San Francisco: Jossey-Bass.

Breslow, L.; Pritchard, D. E.; DeBoer, J.; Stump, G. S.; Ho, A. y Seaton, D. T. (2013). Studying Learning in the Worldwide Classroom Research into edX's First MOOC, Research \& Practice in Assessment, 8, 13-25.

Campbell, J. P., Deblois, P. B. y Oblinger, D. G. (2007). Academic analytics: A new tool for a new era. EDUCAUSE Review, 42(4), 41-57. Recuperado de http://net.educause.edu/ir/library/pdf/ erm0742.pdf

Christensen, C.; Johnson, C. W. y Horn, M. B. (2008). Disrupting Class: How Disruptive Innovation Will Change the Way the World Learns. New York: McGraw-Hill.
Conole, G. (2014, Mayo 14). Good practice in design and delivery of MOOCs [blog post]. Recuperado de http://e4innovation.com/?p=801.

Contact North (2014). Understanding the Building Blocks of Online Learning. Recuperado de http://contactnorth.ca/ sites/default/files/pdfs/understanding the_building_blocks_of_online_learning_2014.pdf

Daniel, J 2012. Making Sense of MOOCs: Musings in a Maze of Myth, Paradox and Possibility. Journal of Interactive Media in Education 2012(3):18, DOI: http://dx.doi.org/10.5334/2012-18 Daniel, J.; Kanwar, A. y Uvalic-Trumbic, S. (2009). Breaking Higher Education's Iron Triangle: Access, Cost, and Quality, Change, march-april, 2009. Recuperado de http://www.changemag. org/archives/back\%20issues/marchapril\%202009/full-iron-triangle.html

Daza, V.; Makriyannis, N. y Rovira Riera, C. (2013). MOOC attack: closing the gap between pre-university and university mathematics, Open Learning: The Journal of Open, Distance and eLearning, 28(3), 227-238

Devlin, K. (2013, Febrero 3). MOOCs and the Myths of Dropout Rates and Certification [blog post]. Recuperado de http://www.huffingtonpost.com/drkeith-devlin/moocs-and-the-myths-ofdr_b_2785808.html

Downes, S. (2013, Abril 4). Digging into MOOC Mania: One Investor's Key Research Questions and Approach [blog post]. Recuperado de http://halfanhour.blogspot.com.es/2013/04/digginginto-mooc-mania-one-investors.html

Feldstein, M. (2014, Octubre 22). Keynote: The Year after the Year of the MOOC [blog post]. Recuperado de 
http://mfeldstein.com/keynote-yearyear-mooc/

Fink, A. (2010). Conducting research literature reviews: From Internet to paper ( $3^{\text {rd }}$ ed.). Thousand Oaks, California: Sage.

Gao, F., Luo, T., y Zhang, K. (2012). Tweeting for learning: A critical analysis of research on microblogging in education published in 2008-2011. British Journal of Educational Technology, 43(5), 783-801.

González-Sanmamed, M.; Muñoz-Carril, P. y Sangrà, A. (2015). Level of Proficiency and Professional Development Needs in Peripheral Online Teaching Roles, The International Review of Research in Open and Distance Learning (IRRODL), 15(6), 162-187.

Guàrdia, L.; Maina, M. y Sangrà, A. (2013). MOOC Design Principles. A Pedagogical Approach from the Learner's Perspective, eLearning $\mathrm{Pa}$ pers, 33. 1-6. Recuperado de http:// www.openeducationeuropa.eu/en/ article/MOOC-Design-Principles.A-Pedagogical-Approach-from-theLearner\%E2\%80\%99s-Perspective

Guo, P. J. y Reinecke, K. (2014). Demographic Differences in How Students Navigate Through MOOCs, L@S 2014 Proceedings of the 1st ACM Conference on Learning at Scale 2014, 21-30.

Haché, P. y Punie, Y. (2012). An exploration of business and sustainability models for OER initiatives in Higher Education. Paper presented at the EADTU 25 th Anniversary Conference. The Role of Open and Flexible Education in European Higher Education Systems for 2020: new models, new markets, new media. Paphos, 27-28 September 2012

Ho, A. D., Reich, J., Nesterko, S., Seaton, D. T., Mullaney, T., Waldo, J. y Chuang, I. (2014). HarvardX and MITx: The first year of open online courses (HarvardX and MITx Working Paper No. 1). Recuperado de http://ssrn.com/ abstract $=2381263$

Hollands, F. M., y Tirthali, D. (2014). MOOCs: Expectations and Reality. Full Report. New York: Teachers College, Columbia University.

Hoxby, C. M. (2014). The Economics of Online Postsecondary Education: MOOCs, Nonselective Education, and Highly Selective Education, NBER Working Paper Series, Working Paper 19816. Cambridge, MA: National Bureau of economic Research. Recuperado de: http://www.nber.org/papers/ w19816

Kizilcec, R. F.; Schneider, E.; Choen, G. L. y McFarland, D. A. (2014). Encouraging Forum Participation in Online Courses with Collectivist, Individualist and Neutral Motivational Framings, eLearning Papers, 37, 13-22.

Lin, M. (2013, Junio 27). Case study: Low participation rate raises question about effectiveness of Massive Open Online Course (MOOC) [blog post]. Recuperado de http://www.mulinblog.com/ case-study-low-participation-rate-raises-question-about-effectiveness-ofmassive-open-online-course-mooc/

Liyanagunawardena, T. R.; Adams, A. A. y Williams, S. A. (2013). MOOCs: A Systematic Study of the Published Literature 2008-2012, The International Review of Research in Open and Distance Learning (IRRODL), 14(3), 202227. Recuperado de http://www.irrodl. org/index.php/irrodl/article/view/1455

Marshall, S. J. (2013). Evaluating the Strategic and Leadership Challenges of MOOCs, MERLOT Journal of Online Learning and Teaching, 9(2), 216-227.

Muñoz-Carril, P.; González-Sanmamed, M. y Hernández-Sellés, N. (2013). Pe- 
dagogical Roles and Competencies of University Teachers Practicing in the E-Learning Environment, The International Review of Research in Open and Distance Learning (IRRODL), 14(3), 462-487.

O'Connor, K. (2014). MOOCs, institutional policy and change dynamics in higher education, Higher Education, 68, 623-635.

O'Shea, T.; Haywood, J.; Woodgate, A.; Conelly, J.; Gormley, S. y Kendra, L. (2013).MOOCs@Edinburgh 2013_Report\#1. Edinburg: University of Edinburgh. Recuperado de http://www.shef. ac.uk/polopoly_fs/1.308890!/file/Edinburgh_MOOCs_Report_2013_1.pdf

Onah, D. F. O.; Sinclair, J. y Boyatt, R. (2014). Drop Out Rates of Massive Open Online Courses: Behavioural Patterns. Edulearn14, $6^{\text {th }}$ International Conference on Education and New Learning Technologies, Barcelona. Recuperado de http://www2.warwick. ac.uk/fac/sci/dcs/people/research/csrmaj/daniel_onah_edulearn14.pdf

Pappano, L. (2012, Noviembre 2). The Year of The MOOC, The New York Times [Articulo electrónico]. Recuperado de http://www.nytimes.com/2012/11/04/ education/edlife/massive-open-onlinecourses-are-multiplying-at-a-rapidpace.html?pagewanted $=$ all\&_r $=0$

Pedreño, A.; Moreno, L.; Ramon, A. y Pernías, P. (2013). La crisis del modelo actual. Los MOOC y la búsqueda de un modelo de negocio, Campus Virtuales, 2(2), 54-65. Recuperado de http://www.revistacampusvirtuales.es/ images/3/4.pdf

Ramírez, M. S. (2014). Guidelines and Success Factors Identified in the First
MOOC in Latin America, Edulearn $14,6^{\text {th }}$ International Conference on Education and New Learning Technologies, Barcelona. Recuperado de http://catedra.ruv. itesm.mx/bitstream/987654321/840/2/ Guidelines $\% 20$ and $\% 20$ success $\% 20$ factors $\% 20$ identified $\% 20$ in $\% 20$ the $\% 20$ first $\% 20$ MOOC $\% 20$ in $\% 20$ Latin $\% 20$ America.pdf

Rivard, R. (2013, Marzo 8). Measuring the MOOC Dropout Rate. Inside Higher Ed [Artículo electrónico]. Recuperado de https://www.insidehighered. com/news/2013/03/08/researchers-explore-who-taking-moocs-and-why-somany-drop-out

Rizvi, S.; Donnelly, K. y Barber, M. (2013). An Avalanche is Coming: Higher Education and the Revolution Ahead. London: Institute for Public Policy Research. Recuperado de http://www. ippr.org/assets/media/images/media/ files/publication/2013/04/avalanche-iscoming_Mar2013_10432.pdf

Rumble, G. (Ed.) (2004). Papers and Debates on the Economics and Costs of Distance and Online Learning. Oldenburg: Bibliotheks- und Informationssystem der Universität Oldenburg.

Schreurs, B.; Van den Beemt, A.; Prinsen, F.; Witthaus, G.: Conole, G. y De Laat, M. (2014). An Investigation into Social Learning Activities by Practitioners in Open Educational Practices, The International Review of Research of Open and Distance Learning (IRRODL), 15(4), 1-20.

Siemens, G. (2004). Connectivism. A Learning Theory for the Digital Age. Elearnspce, 12 Diciembre 2012. Recuperado de http://www.elearnspace.org/ Articles/connectivism.htm 
Siemens, G. (2012, Julio 25). MOOCs are really a platform [blog post]. Recuperado de http://www.elearnspace.org/blog/2012/07/25/moocs-arereally-a-platform/

Stewart, B. L.; Goodson, C. E.; Miertschin, S. L.; Norwood, M. L. y Ezell, S. (2013). Online Student Support Services: A Case Based on Quality Frameworks, MERLOT Journal of Online Learning and Teaching, 9(2), 290-303.

Vargas, J. (2014). What can online course designers learn from research on machine-delivered instruction? Academe, 100(3), 8-12. Recuperado de http://search.proquest.com/docview/15 32082640 ? accountid $=15299$
Veletsianos, G. (2014, Junio 5). The research that MOOCs need [blog post]. Recuperado de http://www.veletsianos. com/2013/06/05/the-research-thatmoocs-need/

Wright, R. (2014, Abril 29). The Death of MOOCs has been greatly exaggerated [Artículo electrónico]. Recuperado de http://www.slate.com/articles/technology/future_tense/2014/04/mooc_completion_rates_don_t_matter.html

Zutshi, S., O'Hare, S. y Rodafinos, A. (2013). Experiences in MOOCs: The Perspectives of Students. American Journal of Distance Education, 27(4), 218-227. 


\section{PERFIL ACADÉMICO Y PROFESIONAL DE LOS AUTORES}

Albert Sangrà, Doctor Europeo en Pedagogía. Profesor agregado de los Estudios de Psicología y Ciencias de la Educación de la Universitat Oberta de Catalunya y Director académico de la Cátedra UNESCO en Educación y Tecnología para el Cambio Social.

Su campo de investigación es el uso de las TIC en educación y la formación y, en particular, las políticas, la organización, la gestión y el liderazgo en la implantación del e-learning y el aseguramiento de la su calidad.

Ha sido director del eLearn Center de la Universitat Oberta de Catalunya y Vicepresidente de la European Foundation for Quality in E-Learning.

Mercedes González-Sanmamed, Doctora en Filosofía y Ciencias de la Educación. Desde 1993 es profesora titular de universidad del área de Didáctica y Organización escolar de la Universidad de A Coruña. Desde 2012 está acreditada como catedrática por la ANECA.

Sus líneas de investigación giran en torno a la formación del profesorado y asesoramiento en educación, integración de las TIC en la educación e innovación y mejora de la escuela.

Ha sido directora del Centro Universitario de Formación e Innovación Educativa de la Universidade da Coruña.

Terry Anderson, Doctor en Psicología Educativa por la Universidad de Alberta, Canadá. Profesor e investigador de la Universidad de Athabasca, en Canadá.

Sus líneas de investigación preferentes se encuentran en el uso de la tecnología para la educación, la educación a distancia y, particularmente, en línea, y las posibilidad de educar grandes grupos de personas mediante modelos colaborativos de aprendizaje.

Es director del Canadian Institute Distance Education Research (CIDER). 
Dirección de los autores: Albert Sangrà

Universitat Oberta de Catalunya

Barcelona Growth Centre

Roco Boronat, 177, 6. ${ }^{\mathrm{a}}$ planta

Barcelona (España)

E-mail: asangra@uoc.edu

Mercedes González-Sanmamed

Universidad de A Coruña

Facultad de Ciencias de la Educación

Campus de Elviña s/n

15071 A Coruña (España)

E-mail: mercedes@udc.es

Terry Anderson

Athabasca University

Peace Hills Trust Tower 1200, 10011 - 109 Street Edmonton, AB

Canada T5J 3S8

E-mail: terrya@athabascau.ca

Fecha Recepción del Artículo: 12. Noviembre. 2014

Fecha Modificación del Artículo: 27. Enero. 2015

Fecha Aceptación del Artículo: 30. Enero 2015

Fecha Revisión para publicación: 30. Enero. 2015 
